\title{
IMMUNOHAEMATOLOGICAL PROFILE OF MATERNAL ANTIBODY-MEDIATED ABO HAEMOLYTIC DISEASE OF FOETUS AND NEWBORN
}

Sajith Vilambili1, Meena Dharmadas², Kumari Krishnakumariamma Chakrapani Usha ${ }^{3}$, Shaiji Panthiyil Shahulhameed ${ }^{4}$, Chitra James ${ }^{5}$, Anjaly Padmavilas Sasikala6, Soonam John7, Vineeth Rajgopal ${ }^{8}$

\begin{abstract}
${ }^{1}$ Assistant Professor, Department of Transfusion Medicine, Government Medical College, Thrissur, Kerala. 2 Professor and HOD, Department of Transfusion Medicine, Government Medical College, Trivandrum, Kerala. 3 Professor and HOD, Department of Transfusion Medicine, Sree Mookambika Institute of Medical Sciences, Kulasekharam, Kanyakumari, Tamilnadu.

${ }^{4}$ Assistant Professor, Department of Transfusion Medicine, Government Medical College, Trivandrum, Kerala. ${ }^{5}$ Assistant Professor, Department of Transfusion Medicine, Government Medical College, Kollam, Kerala. ${ }^{6}$ Senior Resident, Department of Transfusion Medicine, Government Medical College, Thrissur, Kerala. ${ }^{7}$ Assistant Professor, Department of Transfusion Medicine, Government Medical College, Trivandrum, Kerala. ${ }^{8}$ Assistant Professor, Department of Family Medicine, Government Medical College, Calicut, Kerala.
\end{abstract}

\section{BACKGROUND}

\section{ABSTRACT}

The potency of anti-A and anti-B was determined by racial and environmental factors. With respect to ethnic groups, the prevalence and intensity of maternal-newborn $\mathrm{ABO}$ incompatibility varied in different populations.

The aim of this study is to describe the immunological profile of ABO HDFN.

Settings and Design- This was a descriptive study conducted in neonates with ABO HDFN. Research was conducted in Depts. of Transfusion Medicine and Neonatology division of Paediatrics in Govt. Medical College, Trivandrum.

\section{MATERIALS AND METHODS}

Those neonates who fulfilled the inclusion criteria were enrolled in this study. Demographic details, maternal details, bilirubin and haemoglobin levels were recorded. Investigations such as maternal blood grouping and antibody titration were done. In infant's blood sample direct antiglobulin test, blood grouping and elution was performed.

Statistical Analysis- All statistical data were analysed using SPSS software version 16.

\section{RESULTS}

Among mothers of infants with ABO HDFN 25 (22.7\%) had a titre of 128, 34 (30.9\%) had 256, 35 (31.8\%) had 512 and 16 (14.6\%) had 1024. Among 110 infants 34 (30.9\%) were DAT positive, while the rest $76(69.1 \%)$ were DAT negative. While DAT positive was graded $13(11.8 \%)$ fell in grade 1, $16(14.6 \%)$ in grade 2, $3(2.7 \%)$ in grade 3 and $2(1.8 \%)$ in grade 4 categories. Eluate from $59(53.6 \%)$ of infants with ABO HDFN gave a positive result, while the rest $51(46.4 \%)$ gave a negative result. Out of that 59 (53.6\%) elution positive infants, 34 (30.9\%) had a positive DAT and 25 (22.7\%) had a negative DAT; 51 (46.4\%) of infants were negative for both elution and DAT.

\section{CONCLUSION}

Mothers of infants with ABO HDFN had an antibody titre of 128 - 1024. Almost one-third of infants were DAT positive and majority fell in grade 1 or 2 . Eluate from half of infants gave a positive result.

\section{KEYWORDS}

Haemolytic Disease of Foetus and Newborn, Hyperbilirubinaemia, Antibody, ABO, Direct Antiglobulin Test, Elution.

HOW TO CITE THIS ARTICLE: Vilambil S, Dharmadas M, Usha KKC, et al. Immunohaematological profile of maternal antibodymediated ABO haemolytic disease of foetus and newborn. J. Evolution Med. Dent. Sci. 2017;6(72):5107-5112, DOI: $10.14260 /$ jemds/2017/1110

\section{BACKGROUND}

The relative frequency of ABO Haemolytic disease of foetus and newborn (HDFN) varied in different populations. ${ }^{1}$ With respect to ethnic groups, the prevalence of maternalnewborn $\mathrm{ABO}$ incompatibility ranged from $31 \%$ in Europeans

Financial or Other, Competing Interest: None.

Submission 08-07-2017, Peer Review 24-08-2017,

Acceptance 31-08-2017, Published 07-09-2017.

Corresponding Author:

Dr. Sajith Vilambil,

Assistant Professor

Department of Transfusion Medicine,

Government Medical College,

Thrissur-680596, Kerala.

E-mail:drsajithmenon@gmail.com

DOI: $10.14260 /$ jemds $/ 2017 / 1110$ to $50 \%$ in Asians. $^{2}$ Potency of anti-A and anti-B was determined by racial and environmental factors. ${ }^{3}$ This study was to describe the immunological profile of ABO HDFN. Assessing the potent immunogens activity profile was quite an essential tool for implementation of better management protocols.

\section{MATERIALS AND METHODS}

This was a descriptive study conducted on 110 neonates who were suffering from ABO HDFN. Setting for the research was Dept. of Transfusion Medicine and Neonatology division of Sree Avittom Thirunal hospital for women and children attached to Govt. Medical College, Trivandrum. Convenience sampling was done. 
All neonates with hyperbilirubinaemia were admitted in newborn intensive care unit. According to the inclusion criteria, neonates were enrolled in the research. Study was conducted from 01-03-2012 to 30-08-2013.

\section{Inclusion Criteria for ABO HDFN}

1. Raised bilirubin level within first 24 hours of birth.

2. Maternal and neonatal ABO group incompatibility.

3. Similar Rh-D type in mother and baby.

4. Maternal antibody screen negativity.

5. Anti-A or Anti-B IgG titre in maternal serum $\geq 32$.

6. DAT and elution of neonate is positive/elution alone is positive/presence of maternal antibodies in cord blood serum in IAT.

7. No other disease than can be a cause for hyperbilirubinaemia.

\section{Exclusion Criteria}

HDFN in presence of other illnesses like with twin-to-twin transfusion, high output cardiac failure, infection or sepsis, hereditary spherocytosis, glucose-6-phosphate dehydrogenase deficiency, pyruvate kinase deficiency, $\alpha$ Thalassaemia, extravascular blood loss, cephalhaematoma, hypothyroidism, Dubin-Johnson syndrome, cystic fibrosis, biliary atresia, cholestasis, total parenteral nutrition and antibiotic therapy.

Baseline details like name, age, IP no., bed no. and addresses were noted. Bilirubin and haemoglobin levels were recorded.

For classifying hyperbilirubinaemia, chart provided by American Academy of Paediatricians (AAP) was followed. Those coming under high risk and high intermediate risk categories in AAP grading were considered as high bilirubin group and those coming under low intermediate risk and low risk categories were considered as low bilirubin group. Thus, neonates were categorised as low and high bilirubin groups.

Those with haemoglobin $\geq 17 \mathrm{~g} \%$ were categorised as no anaemia and with $<17 \mathrm{~g} \%$ was categorised as anaemic group. The disease was graded as mild, moderate, severe and very severe according to grading proposed by Andrew et al. ${ }^{4}$ (Mild- Grade 0; $\mathrm{Hb}>12.5 \mathrm{~g} / \mathrm{dL}$, no transfusions, ModerateGrade $1: \mathrm{Hb}>12.5 \mathrm{~g} / \mathrm{dL}+$ top-up or exchange transfusion, Severe- Grade $2: \mathrm{Hb}<12.5 \mathrm{~g} / \mathrm{dL}+$ exchange transfusion, Very Severe- Grade 3: intrauterine transfusions and/or $\mathrm{Hb}<10.0$ $\mathrm{g} / \mathrm{dL} \pm$ exchange transfusions or foetal death). Moderate-tosevere disease was considered as one group and mild disease groups was considered as another group in testing association. Duration of stay of neonates in ICU was noted.

$10 \mathrm{~mL}$ venous blood was collected from mothers for doing blood grouping and antibody titration; $5 \mathrm{~mL}$ umbilical cord blood was collected during delivery for performing immuno-haematological investigations.

Determination of ABO group of red cells was performed by tube method. Cell grouping was done using monoclonal anti-A, anti-B and anti-AB. Serum grouping was performed using pooled $A_{1}, B$ and $O$ group cells. Determination of Rh D type of red cells was done with monoclonal IgM anti-D by tube test. Negative test results were tested further for the presence of weak D antigen by an indirect antiglobulin procedure.

Gel cards containing polyspecific antihuman globulin reagent was used for direct and indirect antiglobulin tests.
Using $0.01 \mathrm{M}$ Dithiothreitol (DTT), IgM antibodies was distinguished from IgG antibodies. While considering the titre of antibodies in mothers of infants with ABO HDFN, only the antibody corresponding to the antigen present in the infant was studied. Heat elution procedure was performed in suspected cases of $\mathrm{ABO}$ haemolytic disease.

\section{Ethics}

Study was approved by Human Ethical Committee and Review Board of Institution. Parents of babies were counselled separately about the study and a written consent was procured from them.

\section{Statistical Analysis}

Data analysis was performed in SPSS software version 16. Expression of continuous variables was as mean \pm standard deviation. Qualitative data was being expressed as frequencies and percentages. Comparison of categorical variables were done using chi-square test. $P$ values were two tailed. $\mathrm{P}$ values $<0.05$ were considered statistically significant.

\section{RESULTS}

While considering the titre of antibodies in mothers of infants with ABO HDFN, only the antibody corresponding to the antigen present in the infant was studied. Titre of anti-A was detected in mother of an infant with blood group A and titre of anti-B was detected in mother of an infant with blood group B. Among mothers of infants with ABO HDFN 25 $(22.7 \%)$ had a titre of $128,34(30.9 \%)$ had $256,35(31.8 \%)$ had 512 and $16(14.6 \%)$ had 1024.

Among 110 infants 34 (30.9\%) were DAT positive, while the rest 76 (69.1\%) were DAT negative. While DAT positive was graded $13(11.8 \%)$ fell in grade 1, $16(14.6 \%)$ in grade 2, $3(2.7 \%)$ in grade 3 and $2(1.8 \%)$ in grade 4 categories.

\begin{tabular}{|c|c|c|}
\hline \multirow{2}{*}{ Grades of DAT } & \multicolumn{2}{|c|}{ ABO HDFN } \\
\hline & Frequency & Percentage \\
\hline Negative & 76 & 69.1 \\
\hline 1 & 13 & 11.8 \\
\hline 2 & 16 & 14.6 \\
\hline 3 & 3 & 2.7 \\
\hline 4 & 2 & 1.8 \\
\hline Total & 110 & 100.0 \\
\hline \multicolumn{3}{|c|}{ Table 1. Grading of Results of DAT } \\
\hline
\end{tabular}

Eluate from 59 (53.6\%) of infants with ABO HDFN gave a positive result, while the rest $51(46.4 \%)$ gave a negative result. Out of that $59(53.6 \%)$ elution positive infants, 34 (30.9\%) had a positive DAT and $25(22.7 \%)$ had a negative DAT; 51 (46.4\%) of infants were negative for both elution and DAT.

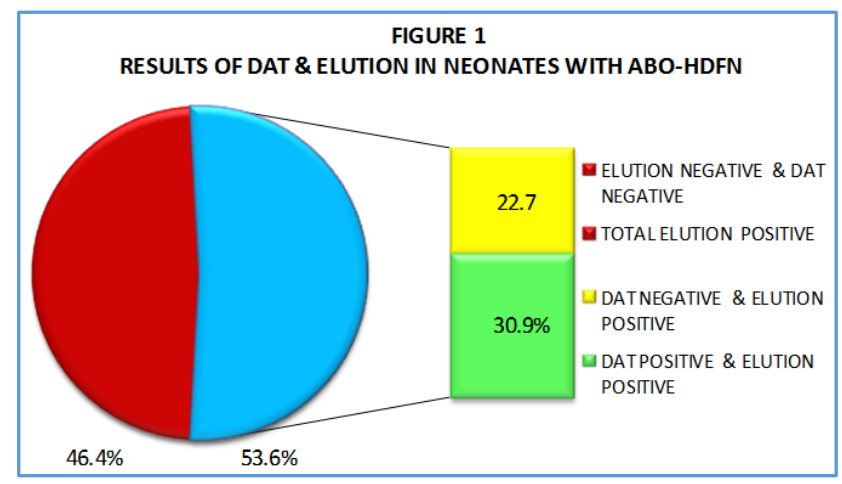


Among 110 neonates $73(66.4 \%)$ belonged to low bilirubin group, $37(33.6 \%)$ to high bilirubin group. Among infants no anaemia was detected in $58(52.7 \%)$ infants and anaemia was detected in 52 (47.3\%); 9 (8.2\%) infants had moderate-to-severe disease and 101 (91.8\%) had mild disease. While considering duration of stay in ICU, 36 $(32.7 \%)$ neonates had a duration of $<1$ week and $74(67.3 \%)$ $\geq 1$ week.

Association between DAT positivity and high bilirubin levels was assessed using chi-square test. $\mathrm{P}$ value was found to be 0.001 , which was significant. DAT positivity had 12.3 times higher risk for high bilirubin levels in neonates affected with ABO HDFN. Odds ratio was 12.294 and confidence interval was $5.692-26.556$.

Association between DAT positivity along with elution positivity and high bilirubin levels was assessed using chisquare test. $\mathrm{P}$ value was found to be 0.001 . DAT positivity along with elution positivity was associated with 4.85 times higher risk for high bilirubin levels in neonates affected with ABO HDFN as compared to DAT negative and elution positive category. Odds ratio was 4.853 and confidence interval was $2.211-10.652$.

Association between DAT positivity and anaemia was assessed using chi-square test and $p$ value was 0.001 . DAT positivity was associated with 3.6 times higher risk for anaemia in neonates affected with ABO HDFN. Odds ratio was 3.576 and confidence interval was 2.432 - 5.259.

On evaluating the association between elution positivity and anaemia by chi-square test, $\mathrm{p}$ value was found to be 0.001 which was significant. Elution positivity was associated with 10.3 times higher risk for anaemia in neonates affected with ABO HDFN. Odds ratio was 10.373 and confidence interval was 4.017 - 26.785 .

Association between DAT positivity and moderate and severe disease was assessed using chi-square test. $\mathrm{P}$ value was 0.004 and it was significant. DAT positivity was associated with 7.8 times higher risk for moderate and severe disease in neonates affected with ABO HDFN. Odds ratio was 7.824 and confidence interval was 1.714 - 35.719 .

Association between elution positivity and moderate and severe disease was assessed using chi-square test. $\mathrm{P}$ value was 0.036 , which was significant. Elution positivity was associated with 7.8 times higher risk for moderate and severe disease in neonates affected with ABO HDFN. Odds ratio was 7.843 and confidence interval was 1.946 - 65.025.

Association of DAT positivity along with elution positivity and moderate and severe disease was assessed using chisquare test. P value was 0.122 , which was not significant. DAT positivity along with elution positivity was not associated with a higher risk for severe disease in neonates affected with ABO HDFN.

Association between DAT positivity and ICU stay for more than one week was assessed using chi-square test. $P$ value was 0.001 , which was significant. DAT positivity was associated with 9.3 times higher risk for stay in ICU in neonates affected with ABO HDFN. Odds ratio was 9.261 and confidence interval was 4.510 - 19.016 .

Association between elution positivity and ICU stay for $>$ 1 week was assessed using chi-square test. $P$ value was 0.001 , which was significant. Elution positivity was associated with 14.7 times higher risk for stay in ICU in neonates affected with ABO HDFN. Odds ratio was 14.695 and confidence interval was 3.712 - 58.176 .

Association between anaemia and ICU stay for more than one week was assessed using chi-square test. $P$ value was 0.001, which was significant. 19 times higher risk of ICU stay for more than one week was noted in anaemic neonates affected with ABO HDFN. Odds ratio was 18.962 and confidence interval was 4.789 - 75.076.

Grades of DAT were positively correlated with risk of peak bilirubin levels, severity of disease and intensity of treatment. Significant negative correlation was seen between grades of DAT and cord blood haemoglobin. Correlation was significant at the 0.01 level.

Antibody titres in mothers of infants with ABO HDFN were positively correlated with grades of DAT, risk of peak bilirubin levels, severity of disease and intensity of treatment. Significant negative correlation was seen between antibody titres in mothers and cord blood haemoglobin. Correlation was significant at the 0.01 level.

\section{DISCUSSION}

The study was conducted in Depts. of Transfusion Medicine and Paediatrics in Govt. Medical College, Thiruvananthapuram from 2012 March to 2013 August; 110 neonates admitted in newborn intensive care unit with $\mathrm{ABO}$ haemolytic disease were included in the study.

Among 110 ABO incompatible mothers 22.7\%, 30.9\%, $31.8 \%$ and $14.6 \%$ had corresponding antibody titres of 128 , 256, 512 and 1024 respectively. In an analysis by Ribera et al, anti-A and anti-B titres in mothers of neonates with $\mathrm{ABO}$ HDFN were higher than $1 / 128$ in $84 \% .^{5}$ Bowley and Voak suggested that an IgG titre of $\geq 256$ in maternal serum had provided a good presumptive evidence of ABO HDFN. ${ }^{6}$ In India, Gupte and Bhatia noticed that all cases of ABO HDFN showed a raised incomplete antibody titre ranging from 1:16 - 1:32. In our investigation, mothers of two severely affected infants with ABO HDFN had a very high titre of antibodies (1024). Likewise, Harvey Klein and David Anstee opined that a titre of 512 or more was found to be very suggestive of severe $\mathrm{ABO}$ haemolytic disease. ${ }^{7}$

Witebsky, Polley MJ et al found out that indirect antiglobulin titre was 1000 or more in $72.2 \%$ cases, in which an infant needed an exchange transfusion. ${ }^{8}$ In an investigation carried out in Kerala, KC Usha and PV Sulochana had observed a direct correlation between high titres of IgM and IgG anti-A or anti-B levels and occurrence of ABO HDFN. ${ }^{9}$

In our study titre of maternal antibody in ABO HDFN was positively correlated with grades of DAT, risk related to peak bilirubin levels, severity of disease and intensity of treatment and was negatively correlated with cord blood haemoglobin levels. A direct correlation between high titres of IgM and IgG anti-A or anti-B levels and ABO HDFN was observed by $\mathrm{KC}$ Usha and Sulochana. ${ }^{9}$

But in contrast to above-mentioned findings, Grundbacher et al and Marsh et al had opined that there existed a poor quantitative relationship between maternal antibody levels and the ensuing haemolytic process, which was attributed to variations in the strength and reactivity of ABO antigens among infants. ${ }^{10,11}$ Chan Shu observed that there was no difference between group A and group B infants in the frequency and severity of haemolytic disease. ${ }^{12}$ 
A slight increase in reticulocytes was a common feature in ABO HDFN and it exceeded $15 \%$ in six out of eleven cases in a research by Crawford et al. ${ }^{13}$ In ABO HDFN, microspherocytes were frequently prominent in blood films and red cell osmotic fragility was almost always above normal limits for upto 2 - 3 weeks after birth. ${ }^{14,15}$

Spin-tube antiglobulin test might be negative in mildly affected infants, while a very sensitive method could demonstrate some anti-A or anti-B on red cells of virtually all ABO incompatible infants. ${ }^{16,17}$ But Harvey Klein and David Anstee has opined that $\mathrm{ABO}$ haemolytic disease neonates might be clinically affected with a negative or only very weakly positive test; DAT might not correctly indicate the amount of antibody bound to the red cells in vivo. ${ }^{7}$

Brouwers et al observed that even in severe $\mathrm{ABO}$ haemolytic disease, the infant's red cells did not react with anti-C3d because of weak expression of A and B antigens on the red cells and relatively low level of complement in the newborn serum. ${ }^{18}$ Voak D and Williams MA opined that eluate from the infant's red cells with weak positive or negative DAT might give strong indirect antiglobulin reactions with adult A1 red cells. ${ }^{19}$

In this study, DAT was positive in $30.9 \%$ of neonates. Few researchers had opined that the DAT was positive in $13 \%$ $40 \%$ of infants with ABO HDFN.18,20,15,21,22,23 Percentage differences in various studies could be due to differences in technique employed for DAT testing, alterations in immunogenicity of A and $\mathrm{B}$ antigens in diverse ethnic groups and dissimilarities in diagnostic criteria for ABO HDFN.

DAT positivity in our study was slightly higher in A group neonates as compared to B group neonates. Peevy and Wiseman inferred in contrast to this finding. ${ }^{1}$

Diagnostic applicability of DAT in ABO HDFN has a wide range of controversies. According to Levine et al and Schonitzer et al DAT was neither a tool for diagnosis nor a predictor of severity of disease, while it was found to be beneficial for establishing ABO HDFN by Bel et al, Dufour et al and Whyte et al.24,25,5,26,27 Dinesh had postulated that in infants with clinically significant haemolysis, $80 \%$ had a positive DAT.28

While Bjarte and Morten opined that antepartum and postpartum serologic tests were poor predictors of $\mathrm{ABO}$ haemolytic disease, Umit et al suggested that reticulocyte count, a positive DAT and the presence of a sibling with neonatal jaundice were good predictors for the development of significant hyperbilirubinaemia and severe haemolytic disease. ${ }^{2,29}$ Dufour concluded that positive DAT and strength of reaction in heat eluate could be used as predictors of prognosis. ${ }^{24}$ Whyte et al observed that DAT was positive in all infants who required treatment for haemolytic jaundice. DAT positive children showed evidence of impending haemolytic anaemia or compensated haemolysis in cord or capillary blood, while elution test had no predictive value. ${ }^{26}$

David et al and Quinn et al observed that DAT was neither diagnostic nor predictive of severity in ABO HDFN.27,20 Density of A and B antigens in cord red cells was studied using an immuno-enzymatic assay and no statistically significant association was found between antigen maturity and severity of ABO HDFN.

In our study, DAT was significantly associated with increased risk of high bilirubin levels and anaemia in ABO HDFN. Similar studies by Desiana et al, Umit et al and
Maissels et al had found out that DAT positivity carried an increased risk of high bilirubin levels. ${ }^{21}$ Whyte et al observed that direct antiglobulin test was positive in all infants who required treatment for haemolytic jaundice and only DAT positive children showed evidence of haemolysis in cord or capillary blood.26 In a study by Rosenfield and Ohno, haemoglobin concentration was slightly lower and bilirubin concentration was slightly higher in $\mathrm{ABO}$ incompatible infants as compared to $\mathrm{ABO}$ compatible infants, while haemoglobin concentration was distinctly lower and bilirubin concentration was distinctly higher among 14\% of DAT positive $\mathrm{ABO}$ incompatible infants in comparison with a negative antiglobulin test group.

Confusion and controversies always existed regarding the significance of laboratory investigations employed in evaluation of ABO HDFN and predictive value of DAT on hyperbilirubinaemia and disease severity. A series shown that those infants whose red cells had greatest evidence of sensitisation had the highest bilirubin and lowest haemoglobin levels. ${ }^{23}$ The minimum criteria for the diagnosis of ABO HDFN suggested by Voak et al was serological demonstration of incompatible anti-A/B antibodies in an eluate prepared from the baby red cells accompanied by the clinical observation of jaundice or more rarely pallor due to anaemia in an infant during the first few days of birth. ${ }^{6}$ Levine et al had postulated that cord blood bilirubin is not diagnostic of haemolytic disease, but was moderately predictive of peak bilirubin levels in ABO HDFN.

Bjarte and Mortem opined that only $20 \%$ to $40 \%$ of ABO incompatible pairs demonstrated a positive DAT and significant haemolysis could be observed with a negative or weakly positive DAT. ${ }^{2}$ Dinesh et al had observed that in infants with clinically significant haemolysis, $80 \%$ had a positive DAT. ${ }^{28}$ Kaplan et al reported that DAT positive ABO heterospecificity was associated with increased haemolysis and a higher incidence of hyperbilirubinaemia. ${ }^{30}$ According to Quinn et al, coombs positivity and strong elution positivity might be a helpful predictor of jaundice but not of its severity. ${ }^{20}$ Dufour et al, Schonitzer et al and Bel et al concluded that DAT was a good screening test for ABO HDFN. ${ }^{24,25,5}$ Levine et al opined that DAT was neither diagnostic nor predictive of severity and it did not correlate with cord blood or peak serum bilirubin levels. Umit Sarici et al observed that neonates with a critical serum bilirubin levels of $4 \mathrm{mg} / \mathrm{dL}$ and $6 \mathrm{mg} / \mathrm{dL}$ at sixth hour of life would be at risk of developing significant hyperbilirubinaemia and severe haemolytic disease respectively. ${ }^{22}$ Kaplan et al opined that O-B heterospecificity carried a higher risk for development of hyperbilirubinaemia as compared to O-A counterparts. ${ }^{30}$

In this survey increasing grades of DAT in ABO HDFN were positively correlated with severity of disease, intensity of treatment and risk related to peak bilirubin levels and negatively correlated with umbilical cord haemoglobin. Likewise, Whyte J and Graham H discoursed that cord serum bilirubin concentration in association with DAT results carried a greater predictive value for ABO haemolytic disease. ${ }^{26}$ In contrast, David et al opined that the DAT was neither diagnostic nor predictive of severity, it was negative in $48 \%$ of infants with serum antibody and it did not correlate with cord blood or peak serum bilirubin levels. ${ }^{31}$ Peevy and Wiseman observed that even though a positive 
DAT was commoner in black than in white infants, there was no difference in bilirubin levels or in the need for phototherapy. ${ }^{32}$ In an analysis by Worlledge et al, frequency of ABO HDFN in Nigeria was about $5 \%$ with predominant anti-B lytic activity. ${ }^{33}$

Here, DAT positivity and severity of disease in ABO HDFN were significantly associated with a higher risk of high bilirubin levels. In a scrutiny by Rosenfield and Ohno, haemoglobin concentration was distinctly lower and bilirubin concentration was distinctly higher among $14 \%$ of DAT positive $\mathrm{ABO}$ incompatible infants in comparison with a negative antiglobulin test group.

Here, DAT positivity and severity of disease in ABO HDFN were significantly associated with a higher risk of high bilirubin levels. Likewise, Umit et al had opined that a positive direct antiglobulin test was a good predictor for severe $\mathrm{ABO}$ haemolytic disease. ${ }^{20}$ But in contrast, Bjarte and Morten observed that antepartum and postpartum serologic tests were poor predictors of $\mathrm{ABO}$ haemolytic disease. In current research, DAT positivity and elution positivity together compared to elution positivity single-handedly was not associated with a higher risk for severe disease. Monoghan and Dufour explained that sex, race, gravidity, birth weight and blood type of the infant do not have significant relationships with clinical outcome. ${ }^{24}$

In our study, whenever the DAT was positive, eluate was also positive for corresponding antibody. Bel and Ribera had opined that elution was positive in all direct antiglobulin test positive ABO incompatible newborn. ${ }^{5}$ Similarly, in our exploration of infants with ABO HDFN, all DAT positive samples yielded elution positivity. In our analysis, $53.6 \%$ of infants with ABO HDFN had elution positivity. Even though DAT was negative, eluate demonstrated the presence of antiA or anti-B in $22.7 \%$ of neonates with ABO HDFN. Positive results with elution method were revealed in 35.1\% - 79.2\% in previous studies by other researchers. ${ }^{23}$ Bowley and Voak had identified that eluate from the infant's red cells with weak positive or negative DAT might give strong indirect antiglobulin reactions with adult $\mathrm{A}_{1}$ red cells, because elution causes increase in antibody concentration. ${ }^{6,19}$

In our analysis, concurrent DAT and elution positivity (30.9\%) were significantly associated with 4.8 times amplified risk for higher bilirubin levels when compared to those in which antibody was detected only by elution (22.7\%). Likewise, Dharmayani et al had observed that those with DAT and elution positivity in concert had a 3.2 times higher risk for higher bilirubin levels when compared to elution alone positive group. Consistent with this finding, Quinn et al had specified that positive DAT with elution was useful in predicting the occurrence of jaundice in $\mathrm{ABO}$ HDFN. ${ }^{20,21}$ Dufour concluded that collective positive results of direct antiglobulin test and strength of reaction in a heat eluate predicted high bilirubin levels. ${ }^{24}$ Similar findings were obtained in our study. In contrast, Whyte et al observed that elution test had no predictive value in assessing the requirement of treatment for haemolytic jaundice in infants with ABO HDFN. 26

There were some limitations in assessing the risk and correlation between variables with a limited data. Convenience sampling due to rarity of disease was another limitation. So it is suggested to do long-term analytical studies for assessing risk and correlation.

\section{CONCLUSION}

Mothers of infants with ABO HDFN had an antibody titre of 128 - 1024. Antibody titres in mothers of infants with ABO HDFN were positively correlated with grades of DAT, risk of peak bilirubin levels, severity of disease and intensity of treatment. Significant negative correlation was seen between antibody titres in mothers and cord blood haemoglobin.

Almost one-third of infants were DAT positive and majority fell in grade 1 or 2 . DAT positivity was found to be a risk factor for high bilirubin levels, anaemia, disease severity and duration of stay in ICU. Grades of DAT were positively correlated with risk of peak bilirubin levels, severity of disease and intensity of treatment. Significant negative correlation was seen between grades of DAT and cord blood haemoglobin.

Eluate from half of infants gave a positive result. Elution positivity was found to be a risk factor for anaemia, disease severity and duration of stay in ICU. DAT positivity along with elution positivity was found to be a risk factor for high bilirubin levels.

\section{ACKNOWLEDGEMENT}

We acknowledge Dr. Anupa Lucas, Dr. Rajany Jose and Dr. Reshmi Ramachandran, Assistant Professors in Dept. of Community Medicine, Govt. Medical College, Thrissur, for their unwavering support and perpetual inspiration in this research. We offer our sincerest obligation to Dr. Lalitha Kailas, Professor and HOD in Dept. of Paediatrics, Gokulam Medical College, Trivandrum, and Dr. Shobha Kumar, Professor in Dept. of Paediatrics, Govt. Medical College, Trivandrum, for their scholarly guidance in the course of this work.

\section{REFERENCES}

[1] Peevy KJ, Wiseman HJ. ABO hemolytic disease of the new born: evaluation of management and identification of racial and antigenic factors. Pediatrics 1978;61(3):475-8.

[2] Solheim BG, Grönn M. Hemolytic disease of the fetus and newborn, Rossi's principles of transfusion medicine. $4^{\text {th }}$ edn. Blackwell Publishing Ltd, 2009:4th edn:420.

[3] Klein H, Anstee D. Mollison's blood transfusion in clinical medicine. $11^{\text {th }}$ edn. Blackwell Publishing Ltd, 2005:530.

[4] Hadley AG, Wilkes A, Goodrick J, et al. The ability of the chemiluminescence test to predict clinical outcome and the necessity for amniocenteses in pregnancies at risk of haemolytic disease of the new born. Br J Obstet Gynaecol 1998;105(2):231-4.

[5] Bel Comos J, Ribera Crusafont A, Natal Pujol A, et al. Value of the coombs test in ABO incompatibility. An Esp Pediatr 1991;35(4):248-50.

[6] Voak D, Bowley CC. A detailed serological study on the prediction and diagnosis of $\mathrm{ABO}$ haemolytic disease of the newborn (ABO HD). Vox Sanguinis 1969;17(5):321-48. 
[7] Klein H, Anstee D. Mollison's blood transfusion in clinical medicine. 11 th edn. Blackwell Publishing Ltd, 2005:531.

[8] Polley MJ, Mollison PL, Rose J, et al. A simple serological test for antibodies causing ABO-haemolytic disease of the new born. Lancet 1965;1(7380):291-5.

[9] Usha KK, Sulochana PV. Detection of high risk pregnancies with relation of ABO hemolytic disease of new born. Indian J Paediatrics 1998;65(6):863-5.

[10] Grundbacher FJ. The etiology of ABO hemolytic disease of the new born. Transfusion 1980;20(5):5638.

[11] Marsh WL, Reid ME, Kuriyan M, et al. Hemolytic disease in the new born. In: A handbook of clinical and laboratory practices in the transfusion of red blood cells. Moneta, VA: Moneta Medical Press, 1993:61-2.

[12] Chan-Shu SY, Blair O. ABO hemolytic disease of the newborn. Am J Clin Pathol 1979;71(6):677-9.

[13] Crawford H, Cutbush M, Mollison PL. Hemolytic disease of the newborn due to anti-A. Blood 1953;8(7):620-39.

[14] Grumbach A, Gasser C. ABO-inkompatibilitäten und morbus haemolyticus neonatorum. Helv Paediatr Acta 1948;3(6):447-74.

[15] Wagle S, Deshpande PG. Hemolytic disease of newborn 2004. emedicine.com

[16] Romano L, Hughes-Jones NC, Mollison PL. Direct antiglobulin reaction in $\mathrm{ABO}$-haemolytic disease of the newborn. Br Med J 1973;1(5852):524-6.

[17] Romans DG, Tilley CA, Dorrington KJ. Monogamous bivalency of IgG antibodies. I. Deficiency of branched ABHI-active oligosaccharide chains on red cells of infants causes the weak antiglobulin reactions in hemolytic disease of the newborn due to $\mathrm{ABO}$ incompatibility. J Immunol 1980;124(6):2807-11.

[18] Brouwers HA, Overbeeke MA, Huiskes E, et al. Complement is not activated in ABO-haemolytic disease of the newborn. $\mathrm{Br} \mathrm{J}$ Haematol 1988;68(3):363-6.

[19] Voak D, Williams MA. An explanation of the failure of the direct antiglobulin test to detect erythrocyte sensitization in $\mathrm{ABO}$ haemolytic disease of the newborn and observations on pinocytosis of IgG anti-a antibodies in infant (cord) red cells. Br J Haematol 1971;20(1):9-23.

[20] Quinn MW, Weindling AM, Davidson DC. Does ABO incompatibility matter? Arch Dis Child 1988;63(10):1258-60.
[21] Dharmayani D, Gatot D, Rohsiswatmo R, et al. Serological profile and hemolytic disease in term neonates with ABO incompatibility. Paediatrica Indonesian 2009;49(4):221.

[22] Ozolek JA, Watchko JF, Mimouni F. Prevalence and lack of clinical significance of blood group incompatibility in mothers with blood type A or B. J Paediatr 1994;125(1):87-91.

[23] Desjardins L, Blajchman MA, Chintu C, et al. The spectrum of $\mathrm{ABO}$ hemolytic disease of the newborn infant. J Pediatr 1979;95(3):447-9.

[24] Dufour DR, Monoghan WP. ABO hemolytic disease of the newborn. A retrospective analysis of 254 cases. Am J Clin Pathol 1980;73(3):369-73.

[25] Schönitzer D, Frisch H. Incidence, laboratory diagnosis and serologic prediction of hemolytic disease of newborn infants due to ABO incompatibility. Padiatr Padol 1984;19(3):263-78.

[26] Whyte J, Graham H. Prediction of the severity of ABO haemolytic disease of the newborn by cord blood tests. Acta Paediatr Scand 1981;70(2):217-22.

[27] Levine DH, Meyer HB. Newborn screening for $A B O$ hemolytic disease. Clin Pediatr 1985;24(7):391-4.

[28] Dinesh D. Review of positive direct antiglobulin tests found on cord blood sampling. J Paediatric Child Health 2005;41(9-10):504-7.

[29] Sarici SU, Yurdakök M, Serdar MA, et al. An early (sixth-hour) serum bilirubin measurement is useful in predicting the development of significant hyperbilirubinemia and severe $\mathrm{ABO}$ hemolytic disease in a selective high-risk population of newborns with ABO incompatibility. Pediatrics 2002;109(4):e53.

[30] Kaplan M, Hammerman C, Vreman HJ, et al. Hemolysis and hyperbilirubinemia in $\mathrm{ABO}$ blood group heterospecific neonates. J Pediatr 2010;157(5):772-7.

[31] Renkonen KO, Timonen S. Factors influencing the immunization of Rh-negative mothers. J Med Genet 1967;4(3):166-8.

[32] Kirkman HN. Further evidence for a racial difference in frequency of $\mathrm{ABO}$ hemolytic disease. J Pediatr 1977;90(5):717-21.

[33] Worlledge S, Ogiemudia SE, Thomas CO, et al. Blood group antigens and antibodies in Nigeria. Ann Trop Med Parasitol 1974;68(3):249-64. 